\title{
ON A DYNAMICAL MODEL OF GLASSES
}

\author{
Jean-Philippe Bouchaud ${ }^{1}$, Alain Comtet ${ }^{2}$ and Cécile Monthus ${ }^{2}$ \\ ${ }^{1}$ Service de Physique de l'Etat Condensé, CEA-Saclay, Orme des Merisiers, 91191 Gif s/ \\ Yvette CEDEX \\ 2 Division de Physique Théorique, IPN, Batiment 100, Université de Paris-Sud, 91406 Orsay \\ CEDEX, and LPTPE, Université P. et M. Curie, 4 Pl. Jussieu, 75231 Paris CEDEX 05.
}

(September 9, 2018)

\begin{abstract}
We analyze a simple dynamical model of glasses, based on the idea that each particle is trapped in a local potential well, which itself evolves due to hopping of neighbouring particles. The glass transition is signalled by the fact that the equilibrium distribution ceases to be normalisable, and dynamics becomes non-stationary. We generically find stretching of the correlation function at low temperatures and a Vogel-Fulcher like behaviour of the terminal time.
\end{abstract}

e-mail addesses

bouchau@amoco.saclay.cea.fr

comtet@ipncls.in2p3.fr

monthus@ipncls.in2p3.fr 
Glasses have a number of fascinatingly universal properties which are still not satisfactorily accounted for theoretically [1], [2]. A common experimental feature is the 'shouldering' of the relaxation laws. More precisely, the relaxation of - say - the density fluctuations evolves from a simple Debye exponential at high temperatures (liquid) to a two-step process at lower temperature, where the correlation function decays fast to a plateau value, from which it subsequently decays on a much longer time scale. These two regimes are called, respectively, the $\beta$ and $\alpha$ relaxations; the $\alpha$ decay is often described in terms of a 'stretched exponential' with a characteristic time scale $\tau$ diverging faster than exponentially with the temperature, and controlling the transport properties such as the viscosity. The most popular description of this divergence is the Vogel-Fulcher law: $\tau \sim \tau_{0} e^{\frac{\Delta}{T-T_{0}}}$, where $\tau_{0}$ is a microscopic time scale [3]. One has to note that despite its tremendous phenomenological success, this law predicts such an abrupt divergence when $T$ is lowered that it cannot be tested near $T_{0}$. Correspondingly, other functional forms, such as $\tau \sim \tau_{0} e^{\left(\frac{\Delta}{T}\right)^{2}}$, give reasonable fits of the data [4], [5]. Furthermore, the Vogel-Fulcher law has only been justified on rather heuristic grounds [6].

Up to now, the most comprehensive theory of dynamical processes in glasses is the socalled mode-coupling theory, developped by Gotze and others [5]. It is based on a family of schematic equations coupling the density fluctuations in a non-linear and retarded way. Generically, these equations have a singularity which is associated to an 'ideal glass' transition temperature $T_{c}$, below which the correlation function does not decay to zero ('broken ergodicity'). This theory describes satisfactorily the overall shape of the relaxation function, at least for $T>T_{c}$ - in particular the existence of the two regimes $\beta$ and $\alpha$ mentionned above, and a power-law divergence of the 'terminal' time scale $\tau$ as $\left(T-T_{c}\right)^{-\gamma}$. However, comparison with experiments [7] shows that the transition temperature $T_{c}$, if it exists, is much higher than the Vogel-Fulcher temperature, leaving a whole temperature interval $\left[T_{0}, T_{c}\right]$ where mode-coupling predicts a 'fluctuation arrest' (i.e. the $\alpha$ regime disappears) while the experimental relaxation time is still finite (and behaves à la Vogel-Fulcher). A way out of this contradiction is to argue that the mode coupling theory leaves out 'acti- 
vated processes' which are responsible for the long time relaxation, and act to blur out the power-law divergence of $\tau$ near $T_{c}$. Although not unconceivable, this possibility requires the introduction of at least one extra free parameter to fit the data, which would be zero in the ideal transition scenario and is not found to be small in the experiments. In other words, one major aspect of the mode-coupling theory is the existence of a singular temperature which however does not manifest itself very directly experimentally - in particular, the terminal time $\tau$ does not reveal any accident around $T_{c}[\mathbf{0}]$.

Another - rather more subtle - difficulty associated with the mode-coupling theory is that the dynamical equations are formally identical [8], [9] to those describing exactly some mean field models of spin-glasses [8], [10], where the presence of quenched disorder is assumed from the start. In glasses, however, this quenched disorder must be in some sense 'self-induced'. Although some progress has recently been made to substantiate such a scenario [11], [12], it is not yet clear whether the glassiness found in mode-coupling theories is or not an artefact of the very approximation.

In this paper, we propose and solve a simple model of glasses. Although still rather abstract, we believe that it captures at least part of the physics involved in the glass transition. The shape of the correlation function evolves, as the temperature is decreased, precisely as in experimental glasses - in particular, the terminal time diverges according to the VogelFulcher law. The glass transition is signalled by the fact that the equilibrium distribution ceases to be normalisable; correlatively, as argued in [13], [14], aging effects are present in the glass phase.

The progressive freezing of a liquid can be thought as follows: each particle is in a 'cage', i.e. a potential well of depth $\epsilon$ created by its neighbours, from which it can escape through thermal activation. However, since a priori all particles can move, the (random) potential well trapping any one of them is in fact time dependent, further enhancing the probability of moving. In order to understand the glass transition, one must describe how, in a self-consistent way, all motion ceases. We thus introduce a density of local potential depth $\rho(\epsilon)$, describing the fact that the efficiency of the 'traps' depend on the environment 
[15]. Now, the basic object on which we shall focus is the probability $P(\epsilon, t)$ that a given particle is in a trap of depth $\epsilon$ at time $t$. This probability evolves because a given particle, with rate $\Gamma_{0} \exp -\frac{\epsilon}{T}$, leaves its trap and chooses a new one with weight $\rho(\epsilon)$. Doing so, all the neighbouring 'traps' are affected by the motion which has taken place. In a mean field description, the resulting evolution of $P(\epsilon, t)$ is described by the following equation:

$$
\frac{\partial P(\epsilon, t)}{\partial t}=-\Gamma_{0} \exp -\left(\frac{\epsilon}{T}\right) P(\epsilon, t)+\Gamma(t) \rho(\epsilon)+\Gamma(t) D \frac{\partial}{\partial \epsilon}\left[\rho(\epsilon) \frac{\partial P(\epsilon, t)}{\partial \epsilon}-P(\epsilon, t) \frac{\partial \rho(\epsilon)}{\partial \epsilon}\right]
$$

where $\Gamma(t) \equiv \Gamma_{0}\left\langle\exp -\left(\frac{\epsilon}{T}\right)\right\rangle$ is the average hopping rate $(\langle\ldots\rangle$ means an average over $P(\epsilon, t)$ itself). The two first terms describe the direct effect of leaving a trap, while the third one expresses the fact that every 'hop' induces a small change in all the neighbouring $\epsilon$ 's. Assuming that the transition rate is proportional to the density of final states, the balance equation reads: $\Gamma(t) \int d \epsilon^{\prime} \mathcal{T}\left(\epsilon-\epsilon^{\prime}\right)\left\{P\left(\epsilon^{\prime}, t\right) \rho(\epsilon)-P(\epsilon, t) \rho\left(\epsilon^{\prime}\right)\right\}$. The fact that the change is small, justified in a mean-field limit where the number of neighbours is large, allows one to write this term in a diffusion like fashion, with an effective diffusion constant $D$ proportional to the width of $\mathcal{T}$. With no incidence on the following results, we shall restrict $\epsilon$ to be positive - in line with our trap picture. Eq. (1) is then supplemented by the boundary condition:

$$
\left(\rho(\epsilon) \frac{\partial P(\epsilon, t)}{\partial \epsilon}-P(\epsilon, t) \frac{\partial \rho(\epsilon)}{\partial \epsilon}\right)=0 \quad \text { for } \quad \epsilon=0
$$

which means that $\epsilon=0$ is a 'reflecting' point. In fact, Eqs. (1) and (2) can be taken as a definition of our dynamical model for glasses, which must be supplemented by an initial condition $P(\epsilon, t=0)$.

Immediate properties of Eqs. $(1,2)$ are that:

- $\int_{-\infty}^{+\infty} d \epsilon P(\epsilon, t)$ is a conserved quantity, as it should.

- When $T \rightarrow \infty, \exp -\left(\frac{\epsilon}{T}\right)=1$ and the equilibrium distribution is simply given by $P_{\text {eq }}(\epsilon) \equiv \rho(\epsilon)$, as expected since there is no Boltzmann factor biaising the a priori weights.

Let us now study the equilibrium distribution at finite $T$. Setting $\frac{\partial P_{\mathrm{eq}}(\epsilon)}{\partial t}=0$, one obtains an inhomogoneous Schrodinger equation for $P_{\text {eq }}(\epsilon)$ : 


$$
-D \frac{\partial^{2} P_{\mathrm{eq}}(\epsilon)}{\partial \epsilon^{2}}+\mathcal{V}(\epsilon) P_{\mathrm{eq}}(\epsilon)=1 \quad \mathcal{V}(\epsilon) \equiv \frac{\Gamma_{0} \exp -\left(\frac{\epsilon}{T}\right)+\Gamma D \frac{\partial^{2} \rho(\epsilon)}{\partial \epsilon^{2}}}{\Gamma \rho(\epsilon)}
$$

with the boundary condition Eq. (2). As long as this equation admits a 'bound state', a normalisable $P_{\mathrm{eq}}(\epsilon)$ exists and we shall call the resulting state 'liquid'. However, as the temperature decreases, the effective potential $\mathcal{V}$ tends to push $P_{\text {eq }}(\epsilon)$ towards larger $\epsilon$, and depending on the shape of $\rho(\epsilon)$, an 'extended', non normalisable state may appear - corresponding to a glass phase. It is easy to show that if $\rho(\epsilon)$ decreases slower than exponentially for large $\epsilon$, the bound state ceases to exist as soon as $T<\infty$, while if $\rho(\epsilon)$ decays faster than exponentially, the bound state remains down to $T=0$ (we shall come back to this case below). We shall thus focus on the case where $\rho(\epsilon)$ is a simple exponential: $\rho(\epsilon) \equiv \frac{1}{T_{0}} \exp -\frac{\epsilon}{T_{0}}$, where $T_{0}$ turns out to be the glass transition temperature. The reason for this is quite simple: it is the temperature at which the Boltzmann weighting factor exactly compensates the fact that deep potential wells are a priori extremely rare. Such a scenario is reminiscent of Derrida's random energy model [16], where the transition temperature is also defined by balancing the locally exponential density of states with the Boltzmann factor.

Solving Eq. (3) for $P_{\text {eq }}(\epsilon)$ in this case leads to a well defined equilibrium state for $T>T_{0}$, which reads [17]:

$$
P_{\text {eq }}(\epsilon)=\mathcal{N}\left[K_{\nu}(x) \frac{I_{\nu-1}\left(x_{0}\right)}{K_{\nu-1}\left(x_{0}\right)} \mathcal{K}_{\nu}\left(x_{0}\right)+K_{\nu}(x)\left(\mathcal{I}_{\nu}\left(x_{0}\right)-\mathcal{I}_{\nu}(x)\right)+I_{\nu}(x) \mathcal{K}_{\nu}(x)\right]
$$

where $\nu=\frac{2 T}{T-T_{0}}, x \equiv x_{0} \exp \frac{\epsilon}{\nu T_{0}}$, and $x_{0}=\frac{\nu T_{0}^{3 / 2}}{\sqrt{D}} \frac{\sqrt{\Gamma_{0}}}{\sqrt{\Gamma}} . I_{\nu}$ and $K_{\nu}$ are the Bessel functions of order $\nu$, and $\mathcal{K}_{\nu}(x) \equiv \int_{x}^{\infty} \frac{d u}{u} K_{\nu}(u), \mathcal{I}_{\nu}(x) \equiv \int_{0}^{x} \frac{d u}{u} I_{\nu}(u) . \mathcal{N}$ and $\Gamma$ are fixed by the normalisation of $P_{\mathrm{eq}}(\epsilon)$ and the boundary condition (2), which lead to the following equation:

$$
\frac{D}{\nu^{3} T_{0}^{3}}=\frac{I_{\nu-1}\left(x_{0}\right)}{K_{\nu-1}\left(x_{0}\right)}\left[\mathcal{K}_{\nu}\left(x_{0}\right)\right]^{2}+2 \int_{x_{0}}^{\infty} \frac{d u}{u} I_{\nu}(u) \mathcal{K}_{\nu}(u)
$$

One can check, using the properties of Bessel functions, that this last equation is identically satisfied when $T \rightarrow \infty$, where $\nu=2$ and $\Gamma=\Gamma_{0}$. For $T \rightarrow T_{0}$, on the other hand, we find that the average hopping rate $\Gamma$ vanishes linearly, as $\Gamma_{0} \frac{T-T_{0}}{T}$, which is numerically found to 
be a very good approximation for all temperatures. More interestingly, however, one finds that for $T \rightarrow T_{0}, P_{\text {eq }}(\epsilon)$ decays as $P_{\text {eq }}(\epsilon) \simeq \frac{T-T_{0}}{T T_{0}} \exp -\frac{\epsilon\left(T-T_{0}\right)}{T T_{0}}$ [19], which means that the characteristic energy scale is $\epsilon^{*}=\frac{2 T T_{0}}{T-T_{0}}$.

In order to make contact with experimental observables one must further define a (twotime) correlation function. The simplest one to consider, corresponding to a high dimensional space, is such that only particles which have not moved at all contribute to the correlation, i.e.

$$
C\left(t, t^{\prime}\right)=\int_{0}^{\infty} d \epsilon P\left(\epsilon, t^{\prime}\right) \exp -\left[\Gamma_{0} \exp -\left(\frac{\epsilon}{T}\right)\left(t-t^{\prime}\right)\right]
$$

but other choices, corresponding e.g. to particles hopping on a d-dimensional lattice, are possible [20]. Eq. (6) assumes in particular that the 'width' of the potential wells is zero. In order to be more realistic and take into account the fast vibration of the particles in their 'cages', a simple modification is to multiply $C\left(t, t^{\prime}\right)$ defined in Eq. (6) by a 'Debye-Waller' factor $C_{\beta}\left(t, t^{\prime}\right)=\exp -\frac{q^{2} r^{2}\left(t-t^{\prime}\right)}{2}$, where $q$ is the probing wave vector. $r(t)$ describes a diffusive motion in an harmonic potential well: $r^{2}(t)=\xi_{0}^{2}\left[1-\exp \left(-\frac{t}{\tau_{0}}\right)\right] ; \xi_{0}$ can be thought as the 'size' of the cage, and $\frac{\xi_{0}^{2}}{\tau_{0}}$ of the order of the high temperature (liquid) diffusion constant [21].

For $T>T_{0}$, i.e. when $P_{\text {eq }}(\epsilon)$ exists, the correlation function only depends on the difference $t-t^{\prime}$. One finds that $C(t)$ (defined by Eq. (6)) behaves as

$$
C(t)= \begin{cases}1-\Gamma t & \text { for } t \ll \Gamma^{-1} \\ \left(\Gamma_{0} t\right)^{\frac{T_{0}-T}{T_{0}}} & \text { for } t \gg \tau(T)\end{cases}
$$

where $\tau(T)=\Gamma_{0}^{-1} \exp \left(\frac{T_{0}}{T-T_{0}}\right)$ is the Vogel-Fulcher time, which very naturally appears within the present model (although no diverging length scale is involved). From Eq. (7), and Fig. 1, one sees that $C(t) C_{\beta}(t)$ has precisely the shape observed in most experimental situations, provided one takes into account the Debye-Waller factor $C_{\beta}$ defined above. Note the presence of two characteristic time scales, a short (microscopic) one $\tau_{0}$, corresponding to the cage vibrations ( $\beta$ peak), and a long one $\tau(T)$, corresponding to the $\alpha$ peak; these two time scales separate extremely fast as the temperature is reduced.

When $T<T_{0}$, on the other hand, no normalisable $P_{\text {eq }}(\epsilon)$ can be found, which corresponds to the weak ergodicity breaking situation described in [13], [14], [10]. In this situation, 
$P(\epsilon, t)$ never reaches a stationary limit, but continuously drifts towards larger and larger energies. Time translational invariance is spontaneously broken as $C\left(t, t^{\prime}\right)$ never becomes a function of $t-t^{\prime}$ alone, a situation now referred to as 'aging'. In fact, one can show [18] that $C\left(t_{w}+t, t_{w}\right) \equiv \mathcal{C}\left(\frac{t}{t_{w}}\right)$, with $1-\mathcal{C}(u) \propto u^{1-\frac{T}{T_{0}}}$ for $u \rightarrow 0$ and $\mathcal{C} \propto u^{-\frac{T}{T_{0}}}$ for $u \rightarrow \infty$, precisely as in the 'trap' model studied in $[13]$. This suggests that the difference between the 'quenched' model considered in [13] (corresponding to $D \equiv 0$ in Eq. (1)) and the 'annealed' model considered here is, to some extent, irrelevant.

All the above results are still expected to hold if the density of states $\rho(\epsilon)$ is approximatively exponential below a certain cut-off $\epsilon_{c}$, provided that $T-T_{0}>\frac{2 T T_{0}}{\epsilon_{c}}$. If $\rho(\epsilon)$ decays faster than exponentially, for example as $\rho(\epsilon) \propto \exp -\left(\frac{\epsilon}{\epsilon_{c}}\right)^{2}$, then strictly speaking the glass temperature is pushed down to zero. This choice for $\rho(\epsilon)$ is interesting since it really corresponds to a mean-field limit where the local trap strength is obtained as a sum of contributions from the (large) number of neighbours. The equilibrium distribution $P_{\mathrm{eq}}(\epsilon)$ is then, for large $\epsilon$, given by $\exp \left[\frac{\epsilon}{T}-\left(\frac{\epsilon}{\epsilon_{c}}\right)^{2}\right]$, and the corresponding $C(t)$ exhibits a considerable amount of stretching at low enough temperatures. $C(t)$ is indeed very well fitted by a stretched exponential at intermediate times [22]. The long time fall off of $C(t)$ is in this case given by $C(t) \propto\left(\frac{\tau(T)}{t}\right)^{\mu}$ with $\mu=\left(\frac{T}{\epsilon_{c}}\right)^{2} \log \Gamma_{0} t$. The terminal time $\tau(T)$ diverges as $\exp \frac{\epsilon_{c}^{2}}{T^{2}}$, i.e. much faster than an activated law, and, as mentioned in the introduction, also compatible with the experimental data [4, [5]. The shape of $C(t) C_{\beta}(t)$ is plotted in Fig. 2 for different $\frac{T}{\epsilon_{c}}$. It is, again, very similar to the experimental data. In particular, the $\alpha$ relaxation for different temperatures can be approximatively [23] rescaled onto a unique master curve when plotted as a function of $\frac{t}{\tau(T)}$, as observed in experiments and numerical simulations [24], and predicted by the mode-coupling theory [5].

In conclusion, we have proposed a very idealized model for the glass transition, which we have solved in the high temperature phase. The results are found to reproduce two striking observations: the shouldering and stretching of the correlation and the Vogel-Fulcher like divergence of the terminal time scale. The former feature is usually accounted for by the mode-coupling theory on the basis of a 'phantom' singularity which is removed by exogeneous 
processes, in turn responsible for the finiteness of the terminal time at low temperatures. Our model suggests that the hypothesis of a intermediate temperature transition might not be necessary, although a more detailed comparison with experimental data is obviously desirable.

Acknowledgments: JPB wants to thank M. Adam, C. Alba-Simionescu, A. Barrat, L. Cugliandolo, D. Dean, J. Kurchan, D. Lairez, J. M. Luck, E. Vincent and especially M. Mézard for many inspiring discussions on these subjects.

Figure Captions.

Fig 1: Exponential density of states. Plot of $C(t) C_{\beta}(t)$ versus $\log _{10}\left(\Gamma_{0} t\right)$ for $q \xi_{0}=0.5$, $\tau_{0}=5 \Gamma_{0}^{-1}$, and $\frac{T}{T_{0}}=2 ., 1.1,1.03$.

Fig 2: Gaussian density of states. Plot of $C(t) C_{\beta}(t)$ versus $\log _{10}\left(\Gamma_{0} t\right)$ for $q \xi_{0}=0.5, \tau_{0}=$ $5 \Gamma_{0}^{-1}$, and $\frac{T}{\epsilon_{c}}=0.5,0.25,0.15$. Note that the plateau observed for the lowest temperature eventually decays to zero. 


\section{REFERENCES}

[1] For a review, see the interesting series of papers in Science, 267 (1995) 1924.

[2] 'Liquids, freezing and glass transition', Les Houches 1989, JP Hansen, D. Levesque, J. Zinn-Justin Editors, North Holland, in particular ref. [5]

[3] H. Vogel, Z. Phys. 22 (1921) 645 ; G. S. Fulcher, J. Am. Ceram. Soc., 6 (1925) 339

[4] J.D. Ferry, L.D. Grandine, E.R. Fitzgerald, J. Appl. Phys. 24 (1953) 911, H. Bassler, Phys. Rev. Lett 58 (1987) 767 and refs. therein.

[5] W. Gotze, in ref. [2], p. 403 et sq.

[6] see T. R. Kirkpatrick, D. Thirumalai, P. G. Wolynes in ref. [8]; T. Vilgis, J. Phys. Cond. Matter,2 (1990) 3667, G. Parisi, Slow dynamics in glasses, preprint cond-mat 941115 and 9412034 .

[7] for a enlightening introduction to the experimental controversy, see the series of Comments in Phys. Rev. E: X.C. Zeng, D. Kivelson, G. Tarjus, Phys. Rev. E 50 (1994) 1711, P. K. Dixon, N. Menon, S. R. Nagel, Phys. Rev. E 50 (1994) 1717, H. Z. Cummins, G. Li, Phys. Rev. E 50 (1994) 1720, and references therein, in particular H. Z. Cummins, W.M. Du, M. Fuchs, W. Gotze, S. Hildebrand, A. Latz, G. Li, N.J. Tao, Phys. Rev. E 47 (1993) 4223

[8] T. R. Kirkpatrick, D. Thirumalai, P. G. Wolynes, Phys. Rev. A 40 (1989) 1045 and references therein. See also Th. Nieuwenhuizen, preprint (1995).

[9] J.P. Bouchaud, L. Cugliandolo, J. Kurchan, M. Mézard, in preparation.

[10] L. Cugliandolo, J. Kurchan, Phys. Rev. Lett. 71 (1993) 173, L. Cugliandolo, P. Le Doussal, Large time off-equilibrium dynamics of a particle diffusing in a random potential, preprint cond-mat 9505112. S. Franz, M. Mézard, Europhys. Lett. 26 (1994) 209, Physica A209 (1994) 1 
[11] T. R. Kirkpatrick, D. Thirumalai, J. Phys. A 22 (1989) L149.

[12] J.P. Bouchaud, M. Mézard J. Phys. I France 4 (1994) 1109, E. Marinari, G. Parisi, and F. Ritort, J. Phys. A 27 (1994) 7615 ; J. Phys. A 27 (1994) 7647 , L. F. Cugliandolo, J. Kurchan, G. Parisi, F.Ritort, Phys. Rev. Lett. 74 (1995) 1012 , S. Franz, J. Hertz, Phys. Rev. Lett. 74 (1995) 2114 . See also: F. Ritort, Glassiness in a Model without energy barriers, preprint cond-mat 9504081

[13] J. P. Bouchaud, J. Physique I 2 (1992) 1705, J. P. Bouchaud and D. S. Dean, J. Phys. I (France) 5 (1995) 265

[14] F. Bardou, J. P. Bouchaud, O. Emile, C. Cohen-Tannouji, A. Aspect, Phys. Rev. Lett. 72 (1994) 203

[15] Note that this one-particle density of state can a priori depend on the density of the liquid and the temperature itself.

[16] B. Derrida, Phys. Rev. B 24, (1981) 2613

[17] The problem of the convergence towards this equilibrium state is interesting and will be discussed in [18]

[18] J.P. Bouchaud, A. Comtet, C. Monthus, in preparation.

[19] This simple exponential form is actually a good numerical approximation for all values of $\epsilon$.

[20] see e.g. J.P. Bouchaud, A. Georges, Phys. Rep. 195 (1990) p. 142-143.

[21] This model emerged from discussions with M. Lairez and M. Adam: see D. Lairez, E. Raspaud, M. Adam, J.P. Carton, J.P. Bouchaud, to appear in 'Die Makromol. Chem. Symp.' (1995).

[22] On this point, see, e.g. B. Castaing, J. Souletie, J. Phys. I (France) 1 (1991) 403. 
[23] Note that $\mu(t=\tau(T))$ is indeed independent of temperature.

[24] W. Kob, H.C. Andersen, Phys. Rev. Lett. 73 (1994) 1376 